\title{
Auch das Pankreaskarzinom differenzierter betrachten
}

\author{
Das Pankreaskarzinom hat eine schlechte Prognose. Und lange \\ Zeit hat sich daran auch nichts geändert. Nun kommt Bewegung \\ in die Therapie. Auch, weil man erkannt hat: Pankreaskarzinom ist \\ nicht gleich Pankreaskarzinom.
}

$\mathrm{B}$ is zum Jahr 2010 stieg die Letalität des Pankreaskarzinoms stetig an. Und noch immer sterben die meisten Patienten innerhalb der ersten zwölf Monate nach Diagnosestellung, die Prognose ist also nach wie vor schlecht. Doch Alexander König, Göttingen, verbreitet Zuversicht. Angesichts intensiver Forschungsaktivitäten hegt er die Hoffnung, dass das Pankreaskarzinom die „rote Laterne an andere Tumorentitäten abgeben kann."

\section{Adjuvant: Gemcitabin plus Capecitabin}

Zunächst eine Bestandaufnahme: Zum Zeitpunkt der Diagnosestellung ist das Pankreaskarzinom bereits bei $60-70 \%$ der Patienten metastasiert, bei $15-20 \%$ lokal fortgeschritten. Hier ist eine palliative Versorgung angezeigt. Nur bei einem knappen Fünftel ist der Tumor resektabel und der Patient kann operiert und einer anschließenden adjuvanten oder additiven Chemotherapie zugeführt werden. Bei lokal fortgeschrittenen Tumoren wird laut König allerdings zunehmend die Überführung in einen operablen Zustand diskutiert. Denn die radikale Resektion ist die einzige kurative Option. Nach der Operation ist eine adjuvante Therapie indiziert. Die Patienten würden signifikant länger leben, wenn sie zusätzlich zum bisherigen Standard Gemcitabin Capecitabin erhielten (median 28 vs. 25,5 Monate; $\mathrm{p}=0,032$ ), so König mit Verweis auf Daten der ESPAC-4-Studie [Neoptolemos JP et al. ASCO. 2016;Abstr LBA4006].
Palliation: Bei sehr gutem Zustand des Patienten aggressiv vorgehen Fortschritte gibt es auch in der Palliation. Über viele Jahre war die Monotherapie mit Gemcitabin Standard in der systemischen Chemotherapie. Durch die Einführung der beiden Therapieregimes nab-Paclitaxel plus Gemcitabin und FOLFIRINOX (Folinsäure, 5-Fluorouracil [5-FU], Irinotecan, Oxaliplatin) wurde die Behandlung klinisch deutlich verbessert. Unter einer Therapie mit FOLFIRINOX lasse sich der Tumor bei etwa $30 \%$ der Patienten verkleinern, erläuterte König. Ähnlich sind die Ergebnisse unter der Kombination aus nab-Paclitaxel und Gemcitabin. Beide Regimes seien für die Erstlinientherapie in der Palliation geeignet, so König. Was aber sollte wem gegeben werden? Sind die Patientenperformance und die Organfunktion sehr gut, plädiert König für die aggressivere Chemotherapie mit FOLFIRINOX. Alter per se sei dabei kein Prädiktor für die Entscheidung.

Von der Zugabe des oralen Tyrosinkinasehemmers Erlotinib zu Gemcitabin profitieren nur wenige Patienten. Als prädiktiver Marker für ein Ansprechen gilt hier ein Hautausschlag innerhalb von acht Wochen nach Therapiebeginn.

Auf dem Prüfstand ist derzeit nanoliposomales Irinotecan. In Kombination mit 5-FU/Folinsäure konnte es gegenüber 5-FU/Folinsäure allein das Gesamtüberleben in der Napoli-1-Studie von 4,2 auf 6,1 Monate verlängern [Wang-Gillam A et al. Lancet. 2016; 387(10018):545-57].

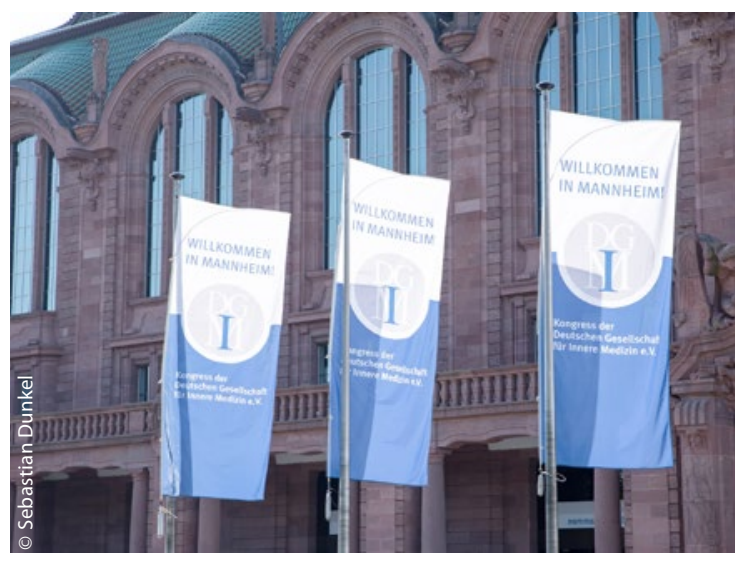

Der Rosengarten in Mannheim war auch in diesem Jahr Tagungsort der DGIM.

\section{"Verhalten optimistisch"}

Die Zukunft in der Therapie des Pankreaskarzinoms sieht König „verhalten optimistisch". Vor allem die bessere molekulare Beschreibung der Tumoren könnte neue Therapiestrategien eröffnen. "Pankreaskarzinom ist nicht gleich Pankreaskarzinom", betonte er. Jeder Tumor hat sein eigenes Profil. Bei Patienten mit BRCA-mutiertem Pankreaskarzinom wird derzeit der PARP(Poly-ADP-Ribose-Polymerase-)Inhibitor Olaparib untersucht, mit ersten günstigen Ergebnissen. Einige wesentliche Fragen sind jedoch noch offen, etwa ob auch Patienten mit somatischen Mutationen (BRCA1/2, PQLB2) im Tumor mit PARP-Inhibitoren behandelt werden können, ob PARPInhibitoren mit platinbasierter Chemotherapie oder mit Topoisomeraseinhibitoren kombiniert werden sollen und ob sich ein PARP-Inhibitor nach Induktionschemotherapie für die Monotherapie im Remissionserhalt eignet. Beate Fessler

Bericht vom Kongress der Deutschen Gesellschaft für Innere Medizin (DGIM) vom 29. April bis 02. Mai 2017 in Mannheim 